\title{
Energy Optimization of Routing Protocols in Wireless Sensor Networks
}

\author{
Fatima Es-sabery*, Hicham Ouchitachen, Abdellatif Hair \\ Faculty of Sciences and Technology, Sultan Moulay Slimane University, B.P. 523, Beni Mellal, Morocco.
}

\begin{tabular}{l} 
Article Info \\
\hline Article history: \\
Received Feb 14, 2017 \\
Revised Jun 26, 2017 \\
Accepted Jul 15, 2017 \\
\hline
\end{tabular}

Keywords:

Hierarchical routing

LEACH

Optimization of energy

PEGASIS

WSNs

\begin{abstract}
The hierarchical routing of data in WSNs is a specific class of routing protocols it encompasses solutions that take a restructuring of the physical network in a logical hierarchy system for the optimization of the consumption of energy. Several hierarchical routing solutions proposed, namely: the protocol LEACH (Low Energy Adaptive Clustering Hierarchy) consist of dividing the network in distributed clusters at one pop in order of faster data delivery and PEGASIS protocol (Power-Efficient Gathering in Sensor Information Systems) which uses the principle of constructing a chain's sensor node. Our contribution consists of a hierarchical routing protocol, which is the minimization of the energy consumption by reducing the transmission distance of data and reducing the data delivery time. Our solution combines the two hierarchical routing approaches: chain based approach and the cluster based approach. Our approach allows for multi-hop communications, intra- and intercluster, and a collaborative aggregation of data in each Cluster, and a collaborative aggregation of data at each sensor node.
\end{abstract}

Copyright (C) 2017 Institute of Advanced Engineering and Science. All rights reserved.

\section{Corresponding Author:}

Fatima Es-sabery,

Laboratory of Applied mathematics and Scientific Calculation,

Faculty of Sciences and Technology, Sultan Moulay Slimane University,

B.P. 523, Beni Mellal, Morocco.

Email: fatima.essabey@gmail.com

\section{INTRODUCTION}

Wireless sensor networks (WSN) consist of a large number of devices known as sensors. These are equipped with the ability to collect physical quantities such as temperature, pressure, $\mathrm{pH}$, etc. in a study area. Then, they perform a processing on the collected data before they cooperate among them to route it to a control center called base station. Due to the small size of the sensors and their low cost of production, WSN offer numerous practical applications; these applications may be sensitive especially in the military, medical, environmental, etc. [1].

Due to the miniaturization constraints [2], the nodes typically have very limited resources in terms of computing capacity, data storage space, transmission and energy flow. These limits are part of the research questions in the field of wireless sensor networks. In particular, the constraint linked to energy is a fundamental problem. Indeed, all elements need energy to operate; the control of energy consumption of a node remains a major problem for maximizing its lifetime [3-5]. Hierarchical routing is considered as a powerful tool as regards to the minimization of the energy consumption compared to other types of routing. Our contribution is to propose a new hybrid approach based on hierarchical protocols [6-9]. 


\section{MODEL OF ENERGY CONSUMPTION}

The sensor node consumes energy to perform three main tasks: detecting, communication and data processing. The energy used for the detection of physical phenomena is not very important. As well as the one used for the treatment is lower than the energy of communication. For example, the necessary energy to transmit $1 \mathrm{~KB}$ over a distance of $100 \mathrm{~m}$ is approximately equivalent to the energy needed to run 3 million instructions with a speed of 100 million instructions per second. While the necessary energy for processing the data is calculated by applying the following formula :

$$
\mathrm{E}_{\mathrm{DA}}=5 \text { nanojoule } / 1 \text { bit }
$$

Since communications dissipate much more energy than other tasks, a power radio's consumption model is proposed by Heinzelman et al. [10] Thus, the necessary energies to emit $E_{t x}$ and receive $E_{r x}$ messages are given by :

To send a message of $\mathrm{k}$ bits over a distance of $\mathrm{d}$ meters, the transmitter consumes:

$$
\begin{aligned}
\mathrm{E}_{\mathrm{tx}}(\mathrm{k}, \mathrm{d}) & =\mathrm{E}_{\mathrm{tx}-\mathrm{elec}}(\mathrm{k})+\mathrm{E}_{\mathrm{tx}-\mathrm{amp}}(\mathrm{k}, \mathrm{d}) \\
& =\mathrm{k} \cdot \mathrm{E}_{\text {elec }}+\mathrm{k} \cdot \mathrm{E}_{\mathrm{fs}} \cdot \mathrm{d}^{2} \text { si } \mathrm{d}<\mathrm{d}_{0} \\
& =\mathrm{k} \cdot \mathrm{E}_{\text {elec }}+\mathrm{k} \cdot \mathrm{E}_{\mathrm{amp}} \cdot \mathrm{d}^{4} \text { si } \mathrm{d} \geq \mathrm{d}_{0}
\end{aligned}
$$

To receive a message of $\mathrm{k}$ bits, the receiver consumes:

$$
\mathrm{E}_{\mathrm{rx}}(\mathrm{k})=\mathrm{k} . \mathrm{E}_{\mathrm{elec}}
$$

Where $E_{t x-e l e c}(k)$ It is the energy transmission, $E_{t x-a m p}(k, d)$ It is the amplification energy $E_{\text {elec }}$ is the amount of energy consumed by a bit and $\mathrm{E}_{\mathrm{fs}}$ is the signal amplification in a lower distance to the threshold distanced $_{0}$. If the distance transmission is superior to $d_{0}$ the amplification $E_{a m p}$ is used Such as:

$$
\mathrm{d}_{0}=\sqrt{\frac{\mathrm{E}_{\mathrm{fs}}}{\mathrm{E}_{\mathrm{amp}}}}
$$

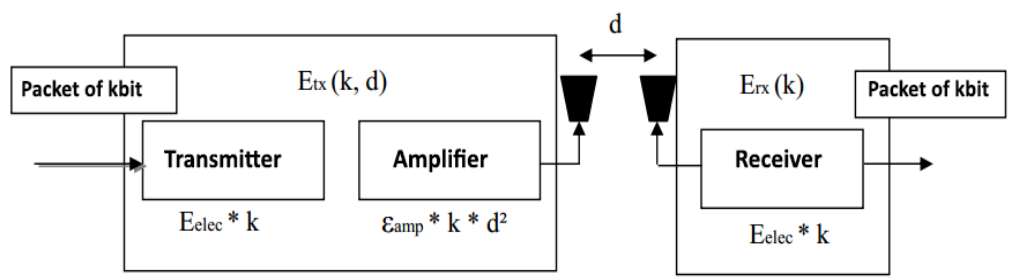

Figure 1. A model of energy consumption

\section{HYBRID APPROACH PROPOSED}

After analyzing the two algorithms (LEACH and PEGASIS) [3,6], we noticed that we can improve the first protocol (LEACH) by applying the concept of the second protocol (PEGASIS) within groups (cluster) and at the level of cluster heads, this leads us to propose a new hybrid protocol which combines the advantages of two broad approaches which are (clustered approach) and (chained approach).

\subsection{Basic Concepts of Our Protocol}

The proposed algorithm consists of combining the two protocols PEGASIS and LEACH according to two major steps:

\section{Step 1: Application of PEGASIS within the cluster}

The organization of the nodes those belong to the same group (cluster) in a chain can improve and regulate the energy dissipation, which reduces the load into cluster-head. Actually, the nodes communicate only with their close neighbors and not directly with their cluster-head, which saves the energetic consumption and offers better use of the bandwidth. The aggregation of data at each node between nodes and cluster-head, this is the consequence of preserving energy reserves in the nodes and cluster-head. 
The figure 2 shows how the nodes are organized in groups (clusters), the C0 node transmits its data to its nearest neighbor $\mathrm{C} 1, \mathrm{C} 1$ aggregates the data received with its own and transmits them to its neighbor until they reach the leader node which transmits them to the $\mathrm{CH}$ (cluster-head). So in this first organizational step (chain group), all nodes in the cluster will transmit their data collected in their respective CHs (clusterheads) by connecting them through the chain, while each $\mathrm{CH}$ receive the collected data by the leader node (the nearest node $\mathrm{CH}$ ) of the chain.
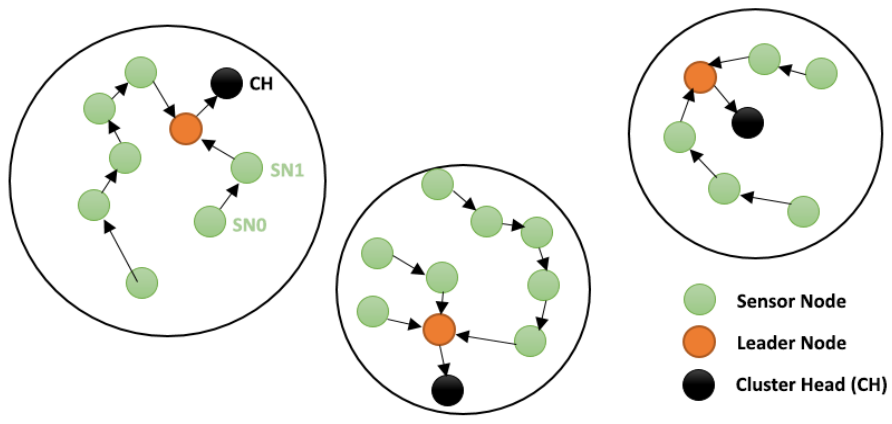

Figure 2. Nodes that are organized in groups (clusters)

Unlike LEACH, the number of nodes that communicate with cluster-head reduced to one node (the leader node). This implies a better energy's save and prolongs the life of the cluster-heads, because if they die (their energy reserve exhausted), all nodes in the cluster will lose their communication with the base station and therefore the entire cluster is considered invalid. In our protocol, we adopted the concept of random rotating of the role of cluster-head proposed by LEACH [14], which regulates the energy dissipation and prevents the nodes chosen as cluster-heads to die quickly. However, in contrast to LEACH, we reused the concept of PEGASIS [2], 14], by organizing cluster nodes as a chain, which has the effect of preventing only the farthest nodes of cluster-heads to exhaust their energy supply.

Concerning the access to the medium (Media Access), we have used the same method proposed in LEACH [14] where cluster-heads establish a transmission plan (TDMA schedule) which assigns to each node the exact time during which it has to transmit its collected data. This allows nodes to turn off their radio antennas and go to the sleepy state, which will help to save more energy. Additionally, TDMA schedule will allow us to avoid the collision and interference problems between cluster nodes.

\section{Step 2: Application of PEGASIS at Cluster heads level}

The principle of this second step is to organize cluster head nodes as a closely neighbor chain. In order to prevent the farthest cluster-heads from the base station to die quickly, thereby, aggregating the data at each cluster-head reduces the number of transmissions to the base station to a single transmission carried out by the cluster-head leader of the chain, which also reduces the load on the base station. This allows to save and to regulate the energy consumption by cluster-heads.

The below figure shows how the cluster-heads nodes will be organized, the node $\mathrm{CH}_{0}$ transmits its data to its nearest neighbor $\mathrm{CH}_{1}, \mathrm{CH}_{1}$ aggregates the data received with its own and transmits them to its neighbor until the cluster-head leader which transmits them to the base station. So in this first organizational step (chain group), all cluster-heads nodes will transmit their collected data respectively to the base station by being connected to the chain, while the base station must receive the data collected by the leader node (the closest node base station) in the chain. 


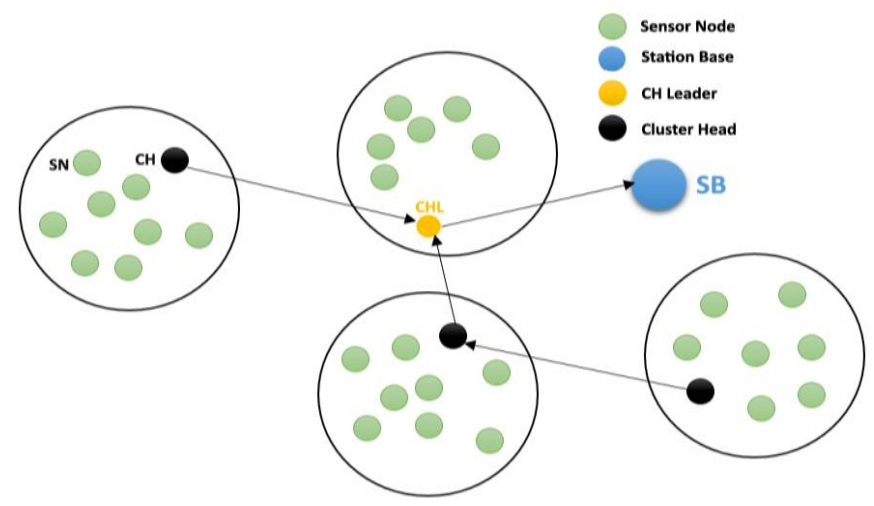

Figure 3. Organization of cluster heads as chain

To summarize, our approach is used to improve the LEACH protocol by using basic concepts of PEGASIS protocol, this improvement can change in the LEACH's topology as shown in the figure below :

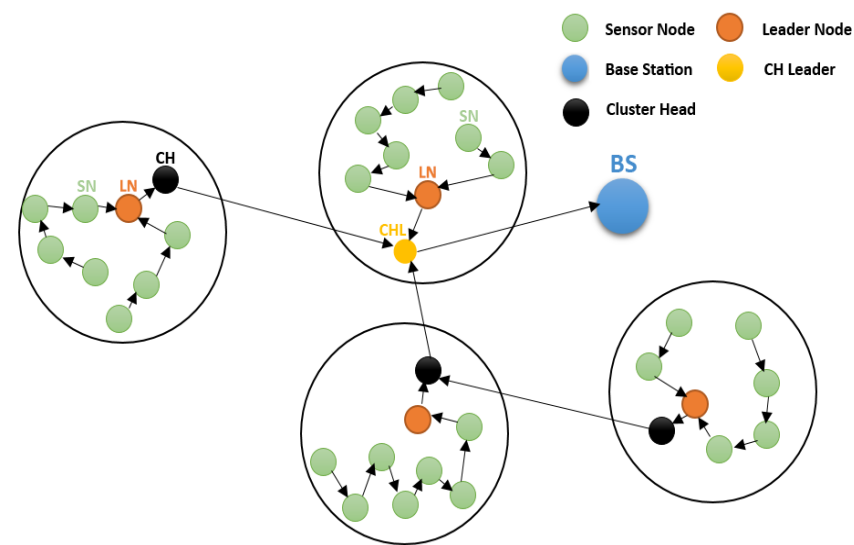

Figure 4. Topology of our hybrid approach

\subsection{Approach of Forming Group in a Chain}

The construction of groups and chains can be performed in a distributed way by cluster-heads (cluster heads) or in a centralized manner by the base station. For best results in terms of an equal distribution of nodes between clusters, we choose the centralized approach proposed by LEACHC protocol [13] where each node sends a data packet to the base station containing the identifier of the node, the reserve energy and the location on the network (using for example the Global Positioning system). The base station will perform an optimization algorithm to form groups (clusters). The optimum cluster determination algorithm is NPcomplex approximation algorithm (NP-HARD), such as the TABOO search algorithm or simulated success algorithm (simulated annealing), which does not give us accurate results but close to optimal.

Therefore, this centralized method of forming a group determines, from the exact position of the nodes, the optimal configuration to minimize energy expended. Once the groups are formed, the base station will switch to the elision of cluster-heads. Those nodes are selected in a very simple manner where only the node that has the largest energy reserve is eligible to become the next head cluster. 


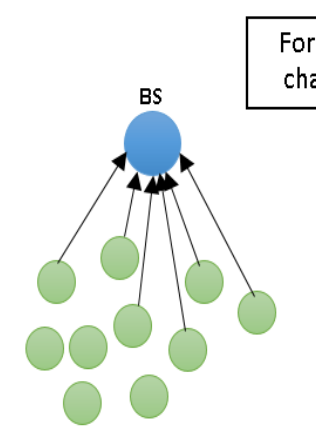

Step 1 : Sends data (id, location and energy level).

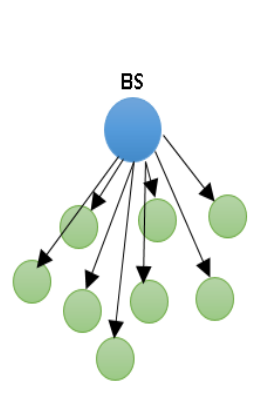

Step 3 : Sends organizational information to all nodes

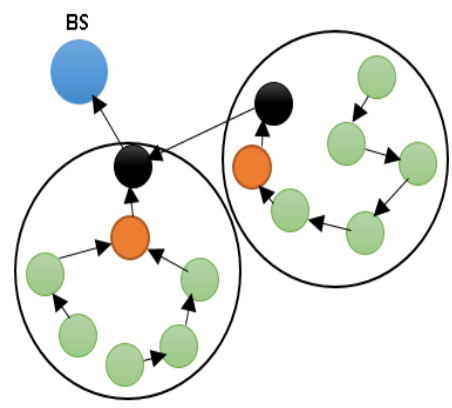

Step 4 : Organization of the network according to our proposed approach

Figure 5. Steps of grouping clusters, as chain by base station

For the formation of channels, we adopted the same idea used in PEGASIS, where nodes of the same group form a chain of closest neighbors for managing intra-cluster communication and cluster-heads nodes form a chain in order to manage inter-cluster communications. The idea is that each node receives data from one of its neighbors, merges (aggregated) data of these with its own data and sends in turn to its other neighbor in the chain. The operation of aggregation is performed at each node in order to eliminate redundant information and reduce the amount of data exchanged for conserving energy.

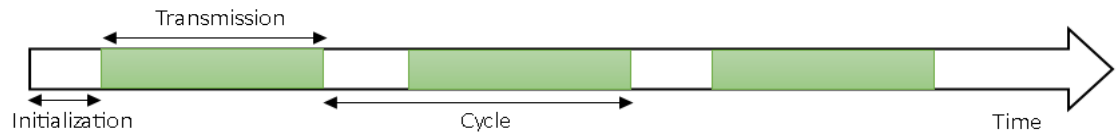

Figure 6. Stages of execution of our hybrid approach

\subsubsection{Initialization Step}

The initialization step begins with the creation of the groups in which we adopt the same approach used in centralized LEACH-C, where the base station uses the simulated success to form groups. This approach provides a better result compared to the distributed approach, used in LEACH, in terms of forming groups and energy conservation. After the formation of groups, cluster-heads are selected in a simplified way where only the node that has the largest reserve of energy among the nodes of the same group, is elected. Then we approach the construction of two chains, chain linked the same cluster member nodes and other nodes linked the cluster-heads where a centralized method is followed in which the base station uses the information sent by the nodes to form chain using the chain forming algorithm proposed by PEGASIS algorithm.

We used the time multiplexing technique as a mean to access to the medium. This technique consists of building a TDMA table to share transmission time on each node. Since each node knows in advance the time slot that will occupy, then it allows the node to switch to the state" asleep" during idle slot. Our approach is to create TDMA table for each group, and will be broadcast to all nodes belonging to it and another TDMA table created at the base station level to manage cluster-heads nodes that form the channel of the closest neighbor for transmitting the data to the base station.

\subsubsection{Transmission Step}

The transmission step is divided into several iterations in which nodes will transmit their collected data, through the chain, to the cluster-heads. In addition, these cluster-heads transmit in turn, their data through the chain they form to the base station. In each iteration, a node transmits at least one data packet during its time slot previously allocated by the base station. Knowing that the time slot allocated to each node is constant, the time for each iteration of transmission will obviously depend on the number of existing nodes in each cluster and the number of cluster-heads.

To reduce energy consumption during data transmission, each node will adjust the power of its radio antenna so that it can only transmit to its nearest neighbors, unlike the LEACH protocol where the farthest 
nodes from cluster-heads and base station lose a lot of energy in order to transmit their data. The use of TDMA schedule created by the base station will allow the nodes the ability to turn off their radio antennas as they wait for their transmission time. This then allows the node to switch to the state "a sleep" during idle slot. Thus, the energy lost due to listening on states (overhearing) and passive listening (idle) is avoided. In contrast to LEACH, the cluster-heads can turn off their antennae until the arrival of the data collected by the chain of nodes and it allows us to conserve more energy.

The transmission phase in our approach is divided into two stages: the first stage concerns the intra_cluster transmission and the second stage concerns the inter-cluster transmission. In the first stage, the members of each cluster node transmit their collected data through the chain to their cluster-head. After the cluster-heads receives data, the transmission process proceeds to the second step of transmitting the collected data by the cluster-heads through the channel to the base station. We can summarize this phase by the following:

a. Data collected through the sensor nodes.

b. Transfer of aggregated and collected data from neighboring nodes through the chain to cluster-heads.

c. The cluster-heads transmit the received data from the closest neighbor through the chain to the base station.

\section{PERFORMANCE EVALUATION OF OUR APPROACH}

The simulation of our hybrid algorithm is the most important stage in our work since we can prove the improvements made in terms of energy savings and overall lifetime of the network using the results provided. The performance analysis of our hybrid routing algorithm is evaluated using MATLAB. The results from the simulation are compared with LEACH algorithms and PEGASIS in terms of network lifetime.

\subsection{Simulation Environment}

In this simulation, our experimental model is built on 100 nodes randomly distributed; the following figure shows a random network of 100 nodes. The base station is located at $(25.150)$ in a field of $50 \mathrm{~m} \times 50 \mathrm{~m}$, and located at $(50,300)$ in a field of $100 \mathrm{~m} \times 100 \mathrm{~m}$. The simulations performed to determine the number of communication rounds when $1 \%, 20 \%, 50 \%$ and $100 \%$ of nodes die in our hybrid protocol. By comparing the result with both LEACH and PEGASIS protocols.

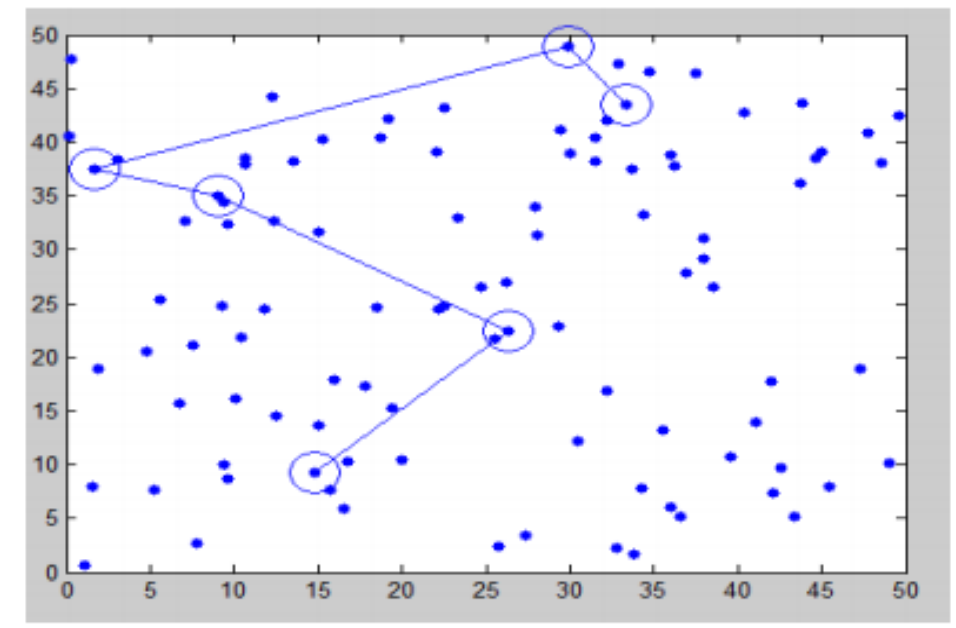

Figure 7. Model of experimentation

We assume that all nodes have a fixed position throughout the simulation period. Our simulation model uses the same parameters mentioned in [14], summarized in the table below: 
Table 1. Simulation parameters

\begin{tabular}{ll}
\hline \multicolumn{1}{c}{ Simulation parameters } & \multicolumn{1}{c}{ Value } \\
\hline Network surface & $50 \mathrm{~m} \times 50 \mathrm{~m} \| 100 \mathrm{~m} \times 100 \mathrm{~m}$ \\
Location of the base station & $(25,150) \|(50,300)$ \\
Number of sensor nodes & 100 \\
Initial energy of the sensor nodes & 0.25 joule $\| 0.5$ joule \\
Number of groups & 5 (because $\mathrm{p}($ Percentage of CHs) $=0.05)$ \\
Data packet size & 2000 bits \\
Electronic energy to transmit a bit & 50njoule / bit \\
Amplification energy & 10njoule $/ \mathrm{bit} / \mathrm{m}^{2} \| 0.0013 \mathrm{pjoule} / \mathrm{bit} / \mathrm{m}^{2}$ \\
Data aggregation energy & 5njoule / bit \\
\hline
\end{tabular}

The base station is set to 100 meters above the nearest node $(X=25, Y=150)$ for a network of $50 \mathrm{~m} \mathrm{x}$ $50 \mathrm{~m}$, the size of a packet of data is 2000 bits. Each node in the network will consume energy in case it will transmit data packets as well as in the case where it reviews data packets also without forgetting the case where it executes data processing (collection and data aggregation). To calculate the energy expended for each sending transaction or receiving data, we used the model of radio energy dissipation proposed in [10].

Initially, all network nodes begin the simulation with an equal energy of $0.25 \mathrm{j}$ and unlimited amount of data to be transmitted to the base station. In addition, the energy of the base station is considered unlimited. Each node uses its limited energy reserve throughout the simulation period, which implies the exhaustion of it. Thus, any node that has exhausted its energy reserve will be considered dead. Therefore, it cannot transmit or receive data.

\subsection{The Results of Simulation}

To compare the lifetime of the network between the two algorithms LEACH and PEGASIS and our algorithm, we measured the residual energy of sensor nodes for each iteration to determine the number of communication rounds when $1 \%, 20 \%, 50 \%$ and $100 \%$ of nodes die, we reused and reconfigured according to our parameters, simulation information LEACH and PEGASIS protocols provided in [10] and [14], and compared with the results of our simulation, the result is given as a graph in below Figures:

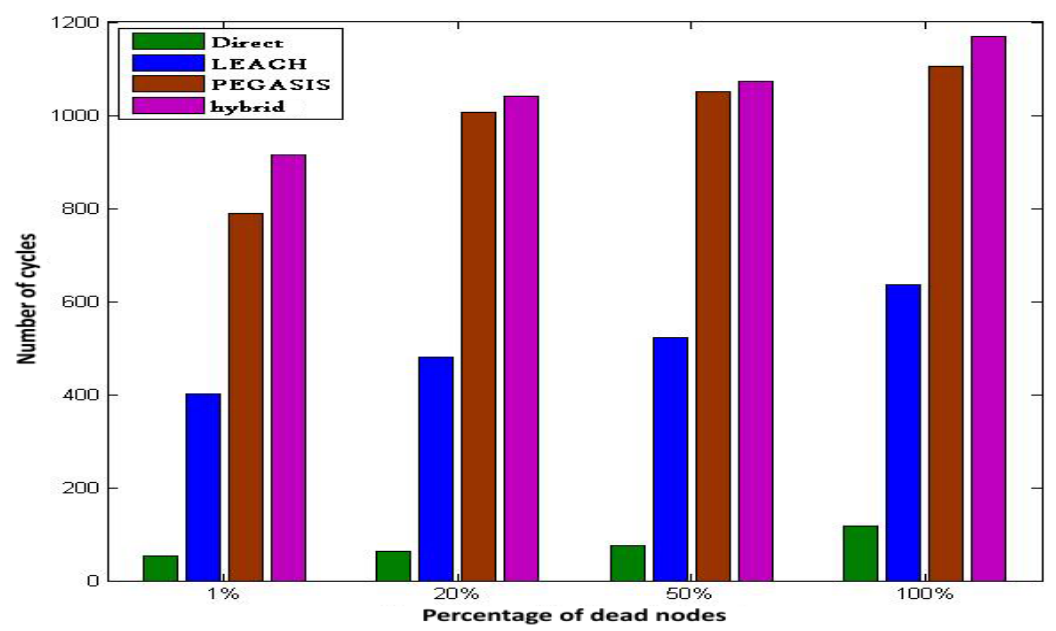

Figure 8 . The performance results of a network of $50 \mathrm{~m} \times 50 \mathrm{~m}$ with an initial energy of $0.25 \mathrm{j} /$ node 


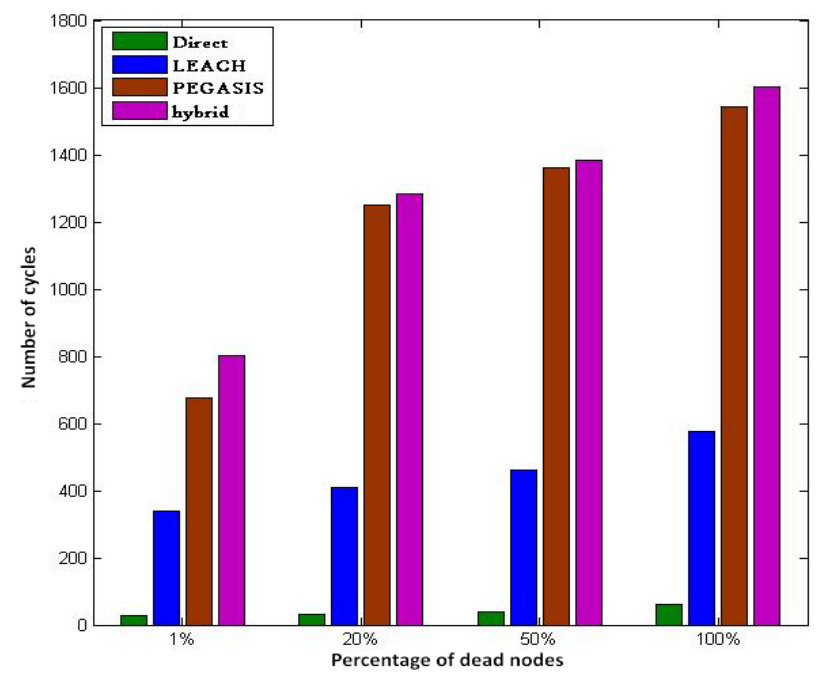

Figure 9. The performance results of a network of $100 \mathrm{~m} \times 100 \mathrm{~m}$ with an initial energy of $0.5 \mathrm{~J} /$ node

Based on simulation results, we have shown that our hybrid algorithm improves the energy dissipation inside and outside clusters, increases energy gain, and extends the lifetime of the network from $50 \%$ to $75 \%$ compared to the LEACH protocol and from $10 \%$ to $17 \%$ compared to PEGASIS protocol.It remains to be noted that our algorithm provided the best value, since it increases the lifetime of the network compared to LEACH protocol, and significantly reduces the extreme latency introduced by the protocol PEGASIS.

\section{COMPARISON OF OUR APPROACH WITH OTHER CLUSTERING PROTOCOLS}

To evaluate the performance of our hybrid algorithm we will compare it with the other clustering protocols such as HEED, TEEN, and LEACH-C using the simulation.

\subsection{Heed Protocol}

O. Younis, S. Fahmy [8] proposed a hierarchical clustering protocol called HEED. This protocol runs in a distributed manner as LEACH, unlike the cluster heads election mode where it uses a formula that involves energy and the cost of communication. Thus, only nodes with higher energy residual may become cluster heads. The HEED algorithm runs in three phases :

Initialization: the algorithm sets a $\mathrm{C}_{\text {prob }}$ threshold to limit the initial number of cluster heads because each network node calculates a probability $\mathrm{CH}_{\text {prob }}$ to become cluster head by the next formula:

$$
\mathrm{CH}_{\text {prob }}=\mathrm{C}_{\text {prob }} * \frac{\mathrm{E}_{\text {residuelle }}}{\mathrm{E}_{\mathrm{Max}}}
$$

where: $\mathrm{E}_{\mathrm{Max}}$ is the initial energy of the node.

$\mathrm{E}_{\text {residuelle }}$ is remaining energy of the node.

$\mathrm{CH}_{\text {prob }}$ is always greater than an inverse threshold $\mathrm{P}_{\min }$.

\subsection{TEEN Protocol}

Manjeshwar and Agrawal [15] proposed a technique called clustering TEEN for critical applications where some change in settings may be abrupt. The network architecture is based on the hierarchical group of several levels where the nearest nodes form clusters. Then this clustering process is passed to the second level until the base station disease [15].

After the forming of clusters, each cluster-head transmits to its members two thresholds: a threshold $\mathrm{H}_{\mathrm{t}}$ (hard threshold), which is the threshold value of the monitored parameter and a threshold $\mathrm{S}_{\mathrm{t}}$ (soft threshold) representing a small variation of controlled parameter value. The occurrence of this small variation St allows the node that detects it, to signal the base station by transmitting a message alert. Therefore, the soft threshold reduces the number of transmissions since it not allows the transmission if there is a little or no change at all in the value of the controlled parameter. 


\subsection{LEACH-C Protocol}

It is a centralized algorithm LEACH protocol, where in the structure of the clusters is calculated at the base station using the optimal method of simulated annealing [13], however, the base station assigns in each iteration of roles for different network nodes (cluster head or single sensor). Then, operation continues in the same manner as LEACH.

Figure 10 shows that our hybrid protocol remains more powerful compared to TEEN, HEED and LEACH-C clustering protocols.

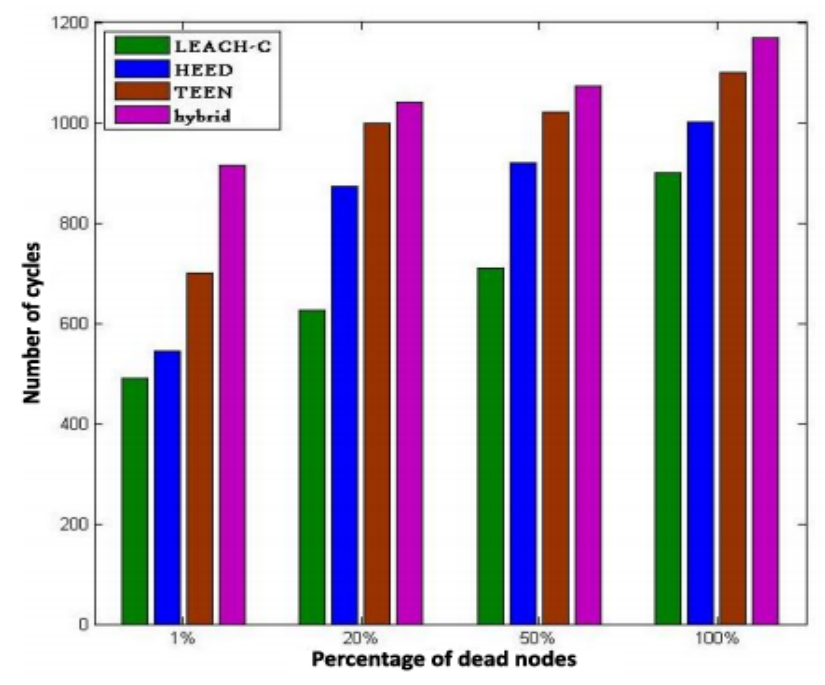

Figure 10. Nodes mortality percentage on the network

\section{CONCLUSION}

Motivated by the extreme latency introduced by the long nodes chain in the PEGASIS protocol and poor energy dissipation in the LEACH protocol, where cluster heads and farthest nodes die faster than others nodes, we tried to propose a new algorithm that combines the benefits of both protocols in order to reduce their disadvantages and provide a best value life / latency. To validate the improvements made by our protocol in terms of extending the network lifetime and the effective management of energy consumption, we simulated the operation of our algorithm with MATLAB and compared the results with those provided protocol LEACH and PEGASIS.

The results from the simulation show that our protocol offers better power management compared to LEACH and PEGASIS protocols. In addition, the degree of latency caused by the long chain in the PEGASIS protocol is significantly reduced.

\section{REFERENCES}

[1] Rajendra Prasad Mahapatra; Rakesh Kumar Yadav, "Descendant of LEACH Based Routing Protocols in Wireless Sensor Networks", vol. 57, pp. 1005-1014, 2015.

[2] Vishal Kumar Arora; Vishal Sharma; Monika Sachdeva, "A Survey on LEACH and other's Routing Protocols in Wireless Sensor Network", vol. 127, pp. 6590-6600, August 2016.

[3] Abbas Nayebi; Hamid Sarbazi-Azad, "Performance Modeling of the LEACH Protocol for Mobile Wireless Sensor Networks", vol. 71, pp. 812-821, June 2011.

[4] Madhura Mahajan; K.T.V. Reddy; Manita Rajput, "Design and Simulation of a Blacklisting Technique for Detection of Hello Flood Attack on LEACH Protocol", vol. 79, pp. 675-682, 2016.

[5] V. Geetha; P.V. Kallapur; Sushma Tellajeera, "Clustering in Wireless Sensor Networks: Performance Comparison of LEACH \& LEACH-C Protocols using NS2,” vol. 4, pp. 163-170, 2012.

[6] Rina Mahakud; Satyanarayan Rath; Minu Samantaray; Baby Sradha Sinha; Priyanka Priya; Ananya Nayak; Aarti Kumari., "Energy Management in Wireless Sensor Network Using PEGASIS", vol. 92, pp. 207-212, 2016.

[7] Young-Long Chen; Jia-Sheng Lin, "Energy Efficiency Analysis of a Chain-Based Scheme via intra-grid for Wireless Sensor Networks”, vol. 35, pp. 507-516, 2012. 
[8] Vishal Kumar Arora; Vishal Sharma; Monika Sachdeva, “A Survey on LEACH and other's Routing Protocols in Wireless Sensor Network”, vol. 127, pp. 6590-6600, 2016.

[9] Abbas Nayebi; Hamid Sarbazi-Azad, "Performance Modeling of the LEACH Protocol for Mobile Wireless Sensor Networks", vol. 71, pp. 812-821, 2011.

[10] Geetha; P.V. Kallapur; Sushma Tellajeera, "Clustering in Wireless Sensor Networks: Performance Comparison of LEACH \& LEACH-C Protocols using NS2”, vol. 4, pp. 163-170, 2012.

[11] Mohammed Omari; Soumia Laroui, "Simulation, Comparison and Analysis of Wireless Sensor Networks Protocols: LEACH, LEACH-C, LEACH-1R, and HEED", pp. 1-5, 2015.

[12] Yaoyue Hu; Jing Liang; Guodong Zhao, "Detection Performances in Radar Sensor Networks using LEACH and HEED”, pp. 2545-2549, 2015.

[13] Jian Shen; Anxi Wang; Chen Wang; Yongjun Ren, Jin Wang, "Performance Comparison of Typical and Improved LEACH Protocols in Wireless Sensor Network", pp. 161-166, 2016.

[14] Binkal S Ahir, Rohan Parmar, Bintu Kadhiwala, "Energy Efficient Clustering Algorithm for Data Aggregation in Wireless Sensor Network", pp. 683-688, 2015.

[15] A. Manjeshwar, D. P. Agrawal, "TEEN: a Routing Protocol for Enhanced Efficiency in Wireless Sensor Networks", pp. 2009-2015, 2001. 